\title{
Contact laser sphincterotomy: further experience and longer follow-up
}

\author{
Inder Perkash \\ Veterans Affairs Palo Alto Health Care System and Stanford University, Stanford, California, USA
}

I report here the use of a crystal chisel contact tip firing Nd:YAG laser for transurethral sphincterotomy in 76 consecutive spinal cord injury patients. Their mean age was 53 years (range 26 to 77 years). Fifty-four (72\%) were complete motor (Frankel A and B) and 21 $(28 \%)$ were incomplete lesions (Frankel C and D) They were evaluated with multichannel urodynamic equipment and $89 \%$ of the patients showed detrusor sphincter dyssynergia and $11 \%$ showed detrusor areflexia. Forty-three patients $(56 \%)$ had previous electrocautery sphincterotomy and were not voiding well. A cystoscopic examination showed that $32 \%$ had an associated enlarged prostate and/or bladder-neck stenosis and $32 \%$ had associated widebody strictures in the bulbous urethra. The crystal chisel contact tip-firing Nd:YAG laser almost mimicked a hot diathermy knife to create an intraurethral incision as well as vaporizing the tissues. For sphincterotomy, a 12 o'clock incision was used from the verumontanum to the bulbous urethra. In patients with an associated enlarged prostate or bulging lateral lobes, 3 and 9 o'clock incisions were also made from the bladder-neck to the verumontanum and also vaporized the bulging prostate tissue. We used 25 to 40 watts for cutting, and vaporization of tissue and 15-25 watts to stop bleeding. The blood loss was less than $50 \mathrm{ml}$ at surgery in $97.4 \%$ patients. None of the patients were transfused. An indwelling Foley catheter was usually left in situ for about $24 \mathrm{~h}$ and the majority of the patients were discharged the next day. All patients have been followed up at least every 6 months for a mean period of 27 months (range 16 to 41 months). The durability of surgery has been checked with linear array transrectal sonography and by urodynamic evaluation. Sixty-nine patients $(92 \%)$ had adequate voiding, minimal to absent autonomic dysreflexia and no significant symptomatic urinary tract infection. There were seven patients who required repeat laser surgery within 2 to 5 months. All subsequent patients are voiding well with wide open bladder-neck and posterior urethra as shown on a voiding cystourethrogram.

Keywords: laser surgery; laser sphincterotomy; urinary bladder; neuropathic bladder; urethral strictures; prostatectomy

\section{Introduction}

Spinal cord injury usually leads to a neuropathic bladder and a neuropathic bowel. Lesions above the conus usually result in an upper motor neuron bladder with reflex voiding. Any damage to the conus or sacral nerve roots results in a lower motor neuron lesion and an areflexic bladder. Patients with supraconal lesions and reflex bladders are not able to empty their bladders due to detrusor-sphincter dyssynergia (DSD). ${ }^{1-3}$ An attempt at voiding can result in a high, sustained intravesical pressure. This can also lead to vesicourethral reflux, repeated pyelonephritis and stone disease. Due to obstructed voiding, this is further complicated with hypertrophy of the bladder or over-distention leading to areflexia of the bladder. Patients with higher lesions (above T5-6) and those with tetraplegia in particular also suffer from autonomic dysreflexia when

Correspondence: Inder Perkash, Professor of Urology \& PVA Professor, Spinal Cord Injury, VA Palo Alto Health Care system (640/128), 3801 Miranda Avenue, Palo Alto, California 94304 they have a full bladder and are unable to void. Intermittent catheterization has now been widely used to empty their bladders. For patients with poor hand function, such as tetraplegic patients, an attendant is needed to perform intermittent catheterization. The majority of such patients ultimately end up with an indwelling catheter which is accompanied by a persistent bladder infection and a higher incidence of stone disease.

Other forms of modalities such as injections of Botulinum-A toxin, ${ }^{4}$ balloon dilation of the sphincter, or placement of an endoluminal urinary sphincter prosthesis $^{2}$ have been used to relieve bladder outflow obstruction due to DSD. These modalities do not address the problem of bladder-bladderneck dyssynergia, the incidence of which seems to be rather high. ${ }^{5,6}$ Therefore, the results have not been very satisfactory to relieve outflow obstruction and also to ameliorate autonomic dysreflexia. ${ }^{7}$ Sacral deafferentation associated with ventral root stimulation has been reported as a promising mode of controlled bladder evacuation 
in selected patients. It seems to be of great value in female SCI patients and in some selected male paraplegic patients. ${ }^{1}$

Transurethral sphincterotomy (TURS) and the use of external condom drainage is now considered a reasonable option to adequately drain the bladder to prevent upper tract changes and loss of kidney function. Transurethral electrosurgical resection of the external sphincter using a diathermy knife has usually been associated with excess blood loss and a high re-operative rate. $^{7}$ In the majority, the failures reported are due to inadequate surgery, post-TURS, strictures, bulbous urethra, and poor detrusor contractility. ${ }^{5,7,8}$

Recently, significant interest has been shown in the use of lasers for endoscopic urological surgery, particularly to ablate an enlarged prostate, instead of the traditional transurethral electro-resection of the prostate (TURP). Following TURP, significant intraoperative and postoperative bleeding, TUR syndrome due to hyponatremia, clot retention, prolonged drainage with a large diameter catheter, urosepsis, urethral strictures and impotence have been reported. The major advantages of laser TUR surgery include minimal blood loss, no risk of hyponatremia and the necessity for a postoperative catheter for only 1 to 2 days following contact laser surgery. ${ }^{8}$

Both contact and non-contact lasers have been used to relieve prostatic obstruction and ablation of urethral strictures. The use of the non-contact beam laser is simple; it relies primarily on thermal coagulation, necrosis and delayed sloughing, which is observed for several weeks after laser beam exposure to the prostatic tissue. Therefore, the majority of patients may require an indwelling catheter drainage for that period of time. The non-contact beam laser has also not been found suitable to cut the bladder neck and external urethral sphincter muscle fibers since the non-contact beam laser works through coagulative necrosis; it might produce melting of muscle fibers and may even have a gluing effect whereupon it could lead to stricture formulation. It is therefore not ideal for ablating urethral strictures and for sphincterotomy.

Both contact and beam lasers are applied through the standard cystoscope and the laser energy is delivered fiberoptically, either through a reusable contact laser probe or through an angled adapter to focus the free beam at the tissues (such as the prostate). Both techniques are used, preferably, under a spinal anesthetic. No tissue is obtained for histology. The usual contact method takes more time than the non-contact technique. Transurethral surgery with the contact laser requires repeated passes to cut and vaporize the tissue to produce a channel; this can take a long time and still not produce an adequate channel. We have developed a technique using a chisel crystal where subsurface 'buttonholes' are created and, following which, ablation of the bridge or overlying tissue is accomplished. ${ }^{8}$ The main advantages of the buttonhole or 'tactilaze' technique, therefore, are a significant control of bleeding and increased vaporization of the tissue. While passing through the tissue, the crystal tip produces hemostasis and excess vaporization, and because of this hemostatic effect the subsequent cutting of the overlying tissue does not produce any bleeding. The tip of the chisel probe cuts the tissue and the side of the probe produces hemostasis. This buttonhole technique creates a larger channel in a shorter period of time when compared with the simple contact repeated passes which only vaporize the surface tissue. Repeated subsurface buttonholes can be created to accomplish an optimal channel in the urethra. This technique is used for prostatic ablation and also for transurethral sphincterotomy.

We have recently reported our early results using a sapphire chisel contact tip-firing $\mathrm{Nd}$ :YAG laser for external sphincterotomy where the blood loss was insignificant and patients were voiding satisfactorily within $1-2$ days following surgery. ${ }^{8}$ There was no incidence of secondary hemorrhage or loss of erection. Herein the technique and results are being reported in 75 consecutive spinal cord injury patients. This will include the patients earlier reported ${ }^{8}$ but now a longer follow-up period is reported to further share our experience and evaluate the durability of the technique.

\section{Methods and materials}

This report relates to 75 spinal cord injury patients with a mean age of 53 years (range 26-77 years) who had contact laser transurethral surgery in the past 41 months. Thirty-five $(46 \%)$ patients were paraplegic and $41(54 \%)$ were tetraplegic. Forty-three $(56 \%)$ had had previous TURS and were not voiding well. Urodynamic studies shaowed detrusor-sphincter dyssynergia in $82 \%$ of the patients. A voiding cystogram showed vesico-ureteral reflex in $32 \%$ of the patients. Cystoscopic examination showed that $32 \%$ had an associated

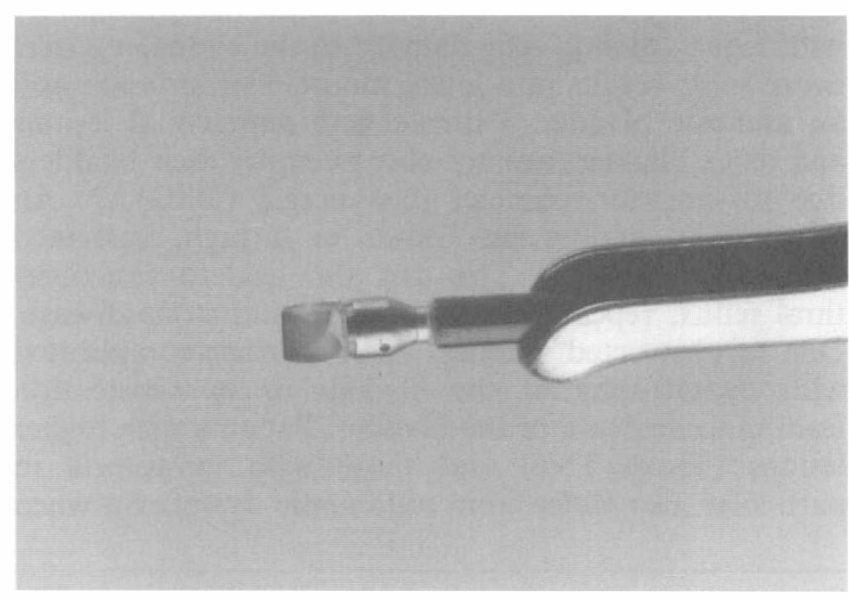

Figure 1 Shows the chisel crystal sapphire screwed onto the tip of a rigid fiber 


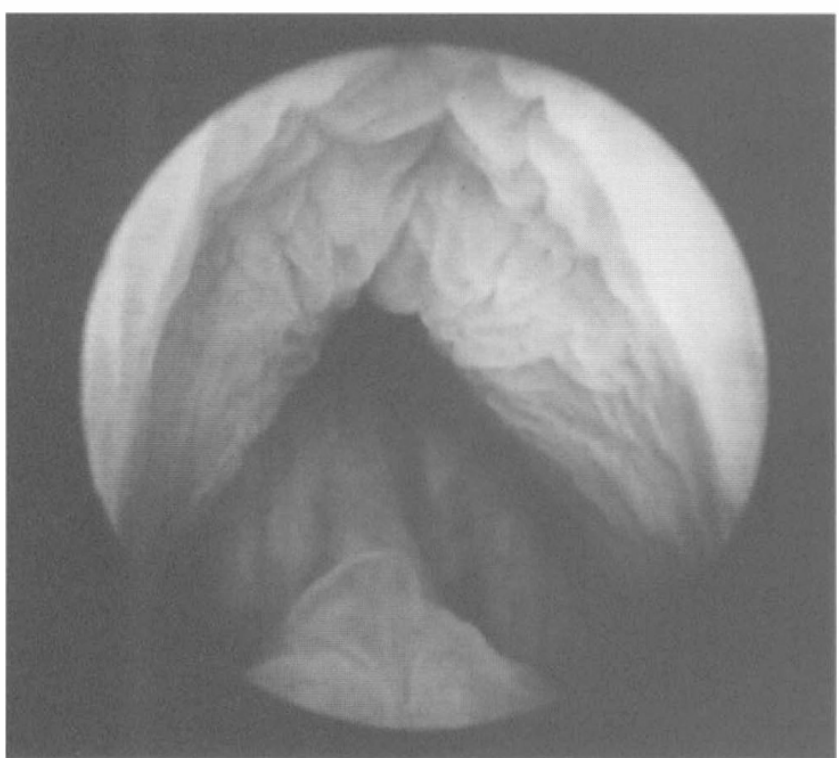

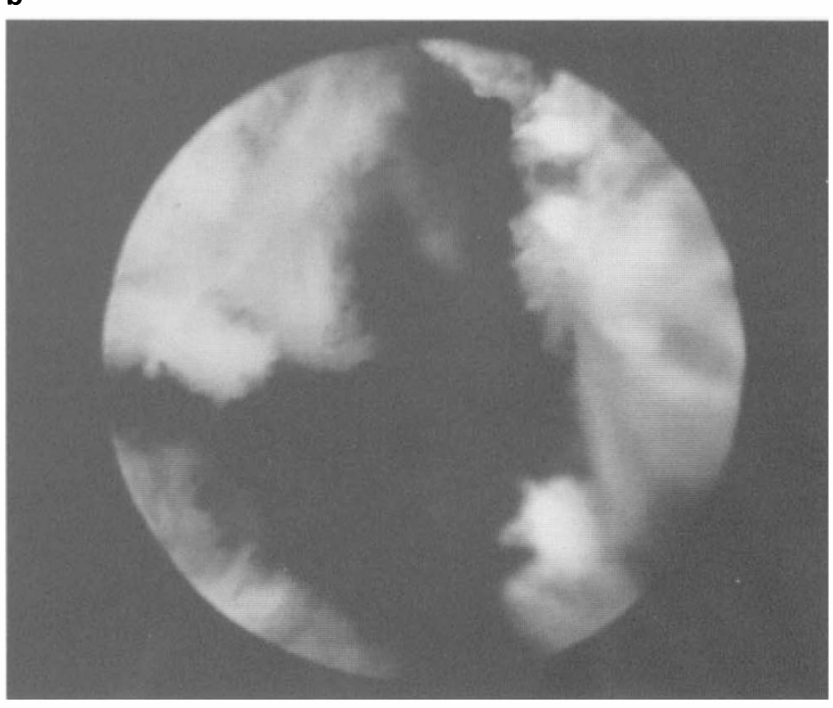

Figure 2 (a) Endoscopy shows prominent bulging mucosa over the external sphincter. (b) Same patient: Endoscopy shows depth of 12 o'clock and 9 o'clock incisions

enlarged prostate and/or bladderneck stenosis, and $32 \%$ an associated stricture in the bulbous urethra.

It is ideal to eradicate urinary tract infection prior to the day of surgery. Most patients were also given a combination of antibiotics about an hour prior to surgery for prophylaxis to cover infection due to gram-negative bacteria. The procedure was performed under a spinal anesthetic in a dorsal lithotomy position. Spinal anesthesia is important to reduce autonomic dysreflexia leading to a rise in blood pressure during surgery.

\section{Surgical technique}

A 21 or 23 French cystoscope with a $30^{\circ}$ lens is used. Urethral landmarks such as the bulbous urethra, verumontanum, lateral prostatic bulge, and the status of the bladder-neck are evaluated. Next, the contact laser system with a chisel probe size $3.5 \mathrm{~mm}$ for 12 o'clock sphincterotomy or a larger chisel probe (Figure 1) was used. The crystal chisel* is screwed onto the tip of the fiber after it has been threaded into the retrograde port of the operating element of the cystoscope. A 12 o'clock sphincterotomy, from the bladder neck to just short of cutting the bulbous urethra, is planned when there is neither raised bladder-neck obstruction nor any excessive bulging of the lateral lobe of the prostate which would also require lateral incisions (TUIP) at 3 and 9 o'clock positions. These incisions are illustrated in Figure 2. Using the contact laser crystal tip, a 12 o'clock incision

*Marketed by SLT, 200 Cresson Boulevard, Oaks, PA 19456-0880 is made by repeated passes over the region. We mostly used 25 to 40 watts of energy for cutting and/or vaporization of tissue and 15 to 25 watts for the stoppage of bleeding. For TURS, a total of about 3000 to 12000 Joules energy (watts/second) was used. If there is excessive fibrosis after a previous surgery (Transurethral sphincterotomy) with the formation of strictures, a higher wattage $(34-50)$ may be needed to cut the fibrosed area. This is a slow process and can take a fair amount of time to vaporize a thickened urethral wall. To improve the speed of vaporization,

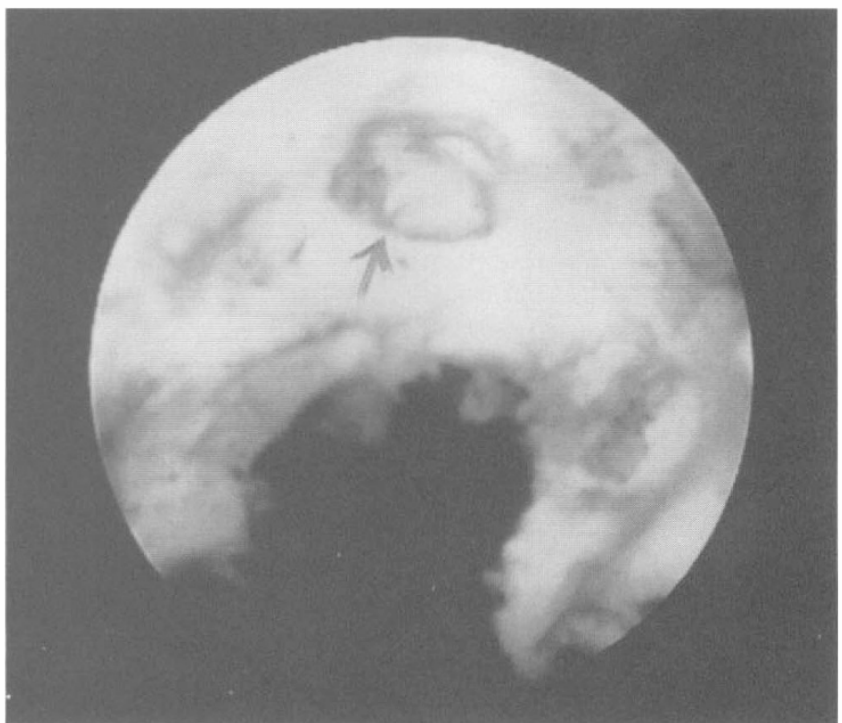

Figure 3 Endoscopy shows creation of a subsurface 'buttonhole' using contact laser to adequately cut urethral sphincter fibers 


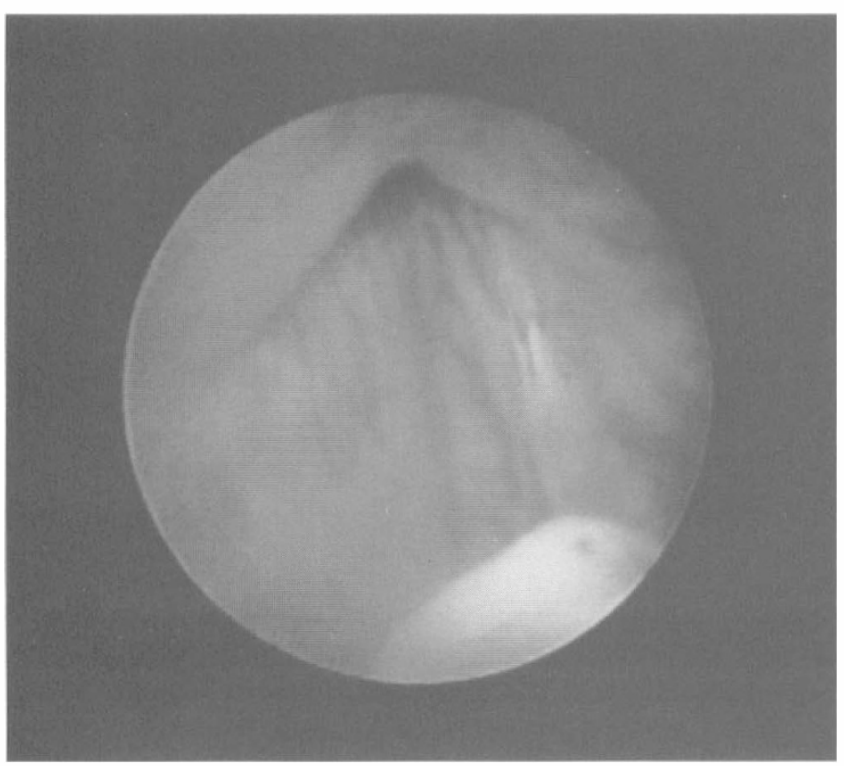

b

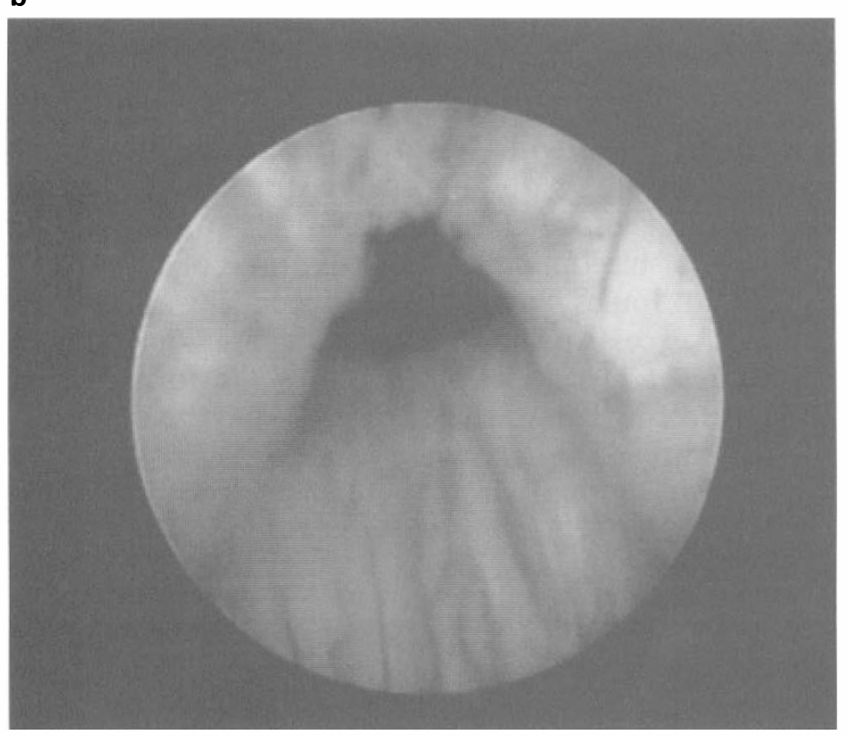

C

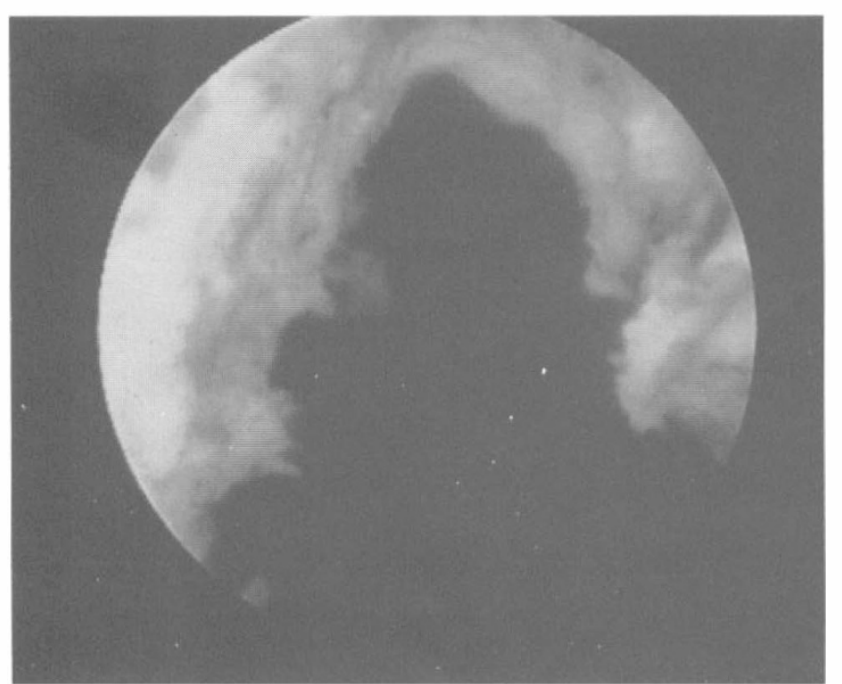

we have developed a technique where, instead of passing the laser crystal on the surface, we make subsurface 'buttonholes' and then divide the overlying tissue by turning the chisel crystal transversely. Attention is paid to push the chisel parallel to the axis of the urethra so that the chisel can ultimately only show up in the bladder and not perforate the urethra. It is preferred to progressively buttonhole small distances of 1 to $2 \mathrm{~cm}$, so that the urethra does not get perforated anteriorly or posteriorly. All fibers are cut and vaporized until the thin fibers of the prostate capsule are disclosed and the sphincter is wide open. At this point, the cystoscope should move freely without any resistance. In an illustrated example, endoscopic examination prior to laser cutting demonstrates an almost closed bladder neck and a prominent verumontanum (Figure 4) and a raised bladder-neck and prominent lateral lobe when fluid is allowed to run from a meter height (Figure 4), and a wide open posterior urethra following a laser incision and vaporization (Figure 4). This case demonstrates associated detrusor bladder-neck dyssynergia along with detrusor sphincter dyssynergia. Sphincterotomy incisions were therefore extended into the bladderneck. To prevent any inadvertent trauma to the rectum, laser incisions and buttonholes are not made below the axis of 3 and 9 o'clock. Any vaporization below 3 and 9 o'clock is carried out by just passing the crystal in a contact mode on the surface of the tissue. Patients with strictures need several passes with the laser to open the urethra. An indwelling catheter is usually left overnight and the patient is generally discharged 24 to $48 \mathrm{~h}$ after surgery.

\section{Postoperative care}

The indwelling catheter, with a balloon distended to $50 \mathrm{ml}$, is usually left in place overnight. In the immediate postoperative period, slight traction (about one pound) is maintained for $4 \mathrm{~h}$. Patients are also given Prazosin $1 \mathrm{mgm}$ orally PRN every 4 to $6 \mathrm{~h}$ if the systolic blood pressure is over $120 \mathrm{mmHg}$ due to autonomic dysreflexia in the immediate postoperative phase, while they have an indwelling Foley catheter. To reduce bladder spasms, we have used Oxybutynin 2.5 to $5.0 \mathrm{mgm}$ twice daily for the first $24-48 \mathrm{~h}$. The Foley indwelling catheter is deflated to $15 \mathrm{ml}$ if dysreflexia extends beyond $4 \mathrm{~h}$. If there is leakage of urine around the catheter, it is removed the same evening. After removal of the catheter, the patient is instructed to gently tap the bladder in the suprapubic region and

Figure 4 (a) Endoscopy shows closed bladder-neck and prominent verumontanum. (b) Same patient: Following infusion at $100 \mathrm{~cm}$ pressure, slightly open bladder-neck which is also raised with prominent lateral bulges. (c) Wide open posterior urethra following laser incision and vaporization 


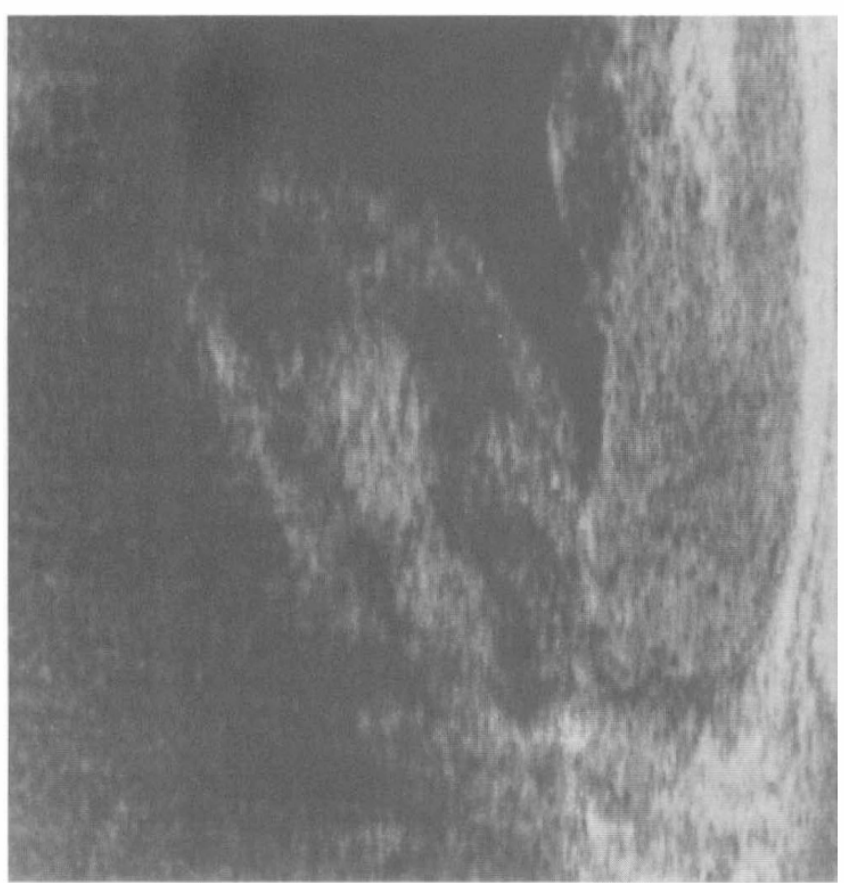

b

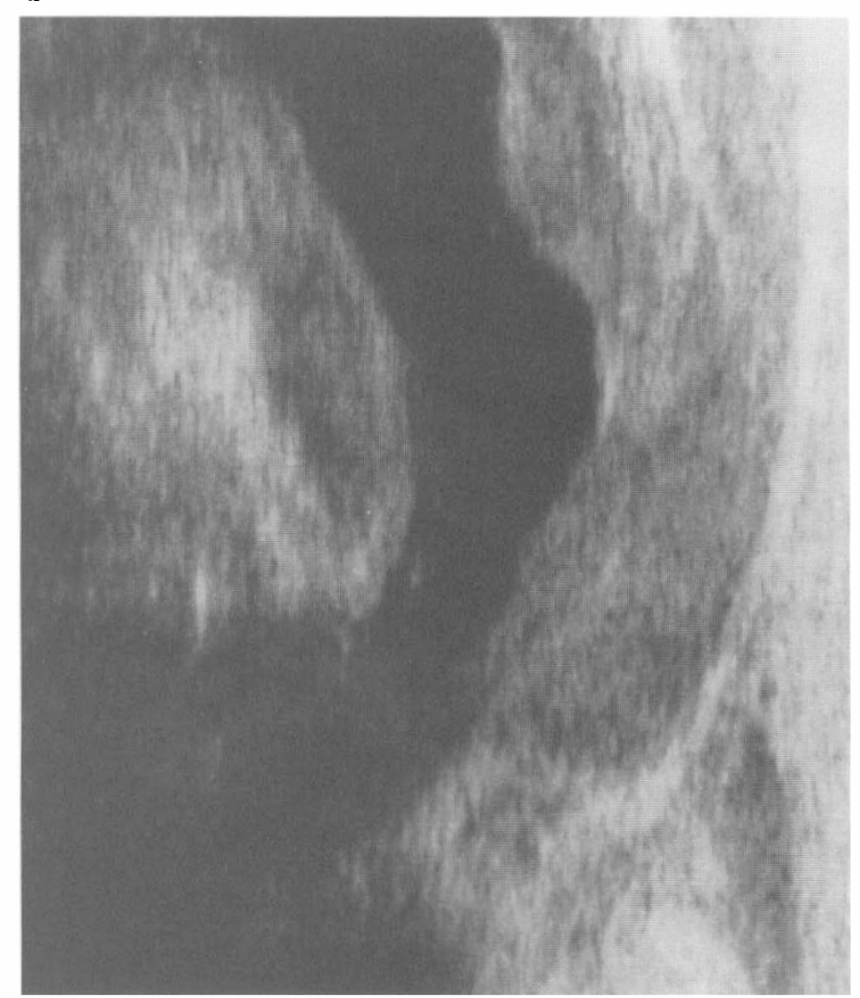

Figure 5 (a) In a tetraplegic patient, transrectal linear array sonographic voiding cystogram shows open bladder-neck but closed external urethral sphincter. (b) Following TURS a year later, wide open urethra from bladder-neck to bulbous urethra

bear down to empty the bladder every $2 \mathrm{~h}$. All patients were followed up for the possible development of urinary tract infection, any episodes of autonomic dysreflexia and to observe the adequacy of voiding and durability of surgery with a transrectal linear array sonographic voiding cystourethrogram. An illustrated example of the follow-up results is shown in Figure 5.

\section{Results}

Intraoperative blood loss was less than $50 \mathrm{ml}$ in $97.4 \%$ of patients. In two patients the blood loss was 150 and $300 \mathrm{ml}$. In one patient where lateral incisions were extended into the bulbous urethra with a higher wattage setting (45 watts) there was excessive bleeding; in the second patient also higher wattage (45 watts) was used for ablation of the prostate. These were the first two patients when we started using the contact laser and were still developing the technique and we did not lower the wattage when trying to stop venous bleeding. No patient was transfused with blood. There was no incidence of secondary hemorrhage. All patients underwent preoperative urodynamics. In the first 20 patients, post-operative urodynamics showed a mean drop in maximum voiding pressures of $37.0 \mathrm{~cm}$ $\mathrm{H}_{2} \mathrm{O}$ (range 4 to $112 \mathrm{~cm}$ ); this was a significant change $(P$ value $<0.0003)$. In three other patients there was a rise of maximum voiding pressure of 4,24 and $39^{\circ} \mathrm{cm}$ respectively following surgery. This was not a sustained rise but a quick rise and drop in voiding pressure. Sixty-nine $(92 \%)$ patients had adequate voiding with minimal to nil features of autonomic dysreflexia and also had no significant urinary tract infection (UTI). Our criteria for significant UTI is less than two symptomatic infections in a 6 month period in spinal cord injury patients. They showed wide open posterior urethra on transrectal sonographic voiding cystography during follow-up. The initial four patients, and subsequently three other patients, needed repeat surgery. In three patients, initial prostatic ablation did not adequately improve their voiding; therefore, TURS was done with subsequent adequate voiding. Two had areflexic bladders, and repeat TURS succeeded in their being able to empty their bladder by credé/valsalva. One patient had inadequate TURS incisions at first surgery which were deepened a second time. Two of the patients also had a scarred bladder neck, and lateral transurethral incisions with laser adequately opened the bladder-neck.

\section{Discussion}

External sphincterotomy has been used to relieve outflow obstruction due to DSD in spinal cord injury patients since 1957. ${ }^{11-13}$ Several modifications in the technique have been tried, including balloon dilation 
and recently a metallic stent has been placed in the posterior urethra to prevent closure of the urethra by a dyssynergic external urethral sphincter. ${ }^{2}$ The long term results are awaited. The stent does not extend into the bladder-neck, therefore obstruction due to bladderbladder-neck dyssynergia still remains; these patients also have a high incidence of autonomic dyssynergia. They may need surgery to cut the bladder-neck and/or the use of alpha blockers to open the bladder-neck and to treat autonomic dysreflexia. In such patients, following bladder-neck incisions, there is a potential danger of the stent migrating into the bladder.

Following electrocautery surgery (TURS), intraoperative and secondary bleeding have been the major complications. There has also been a significant failure rate, ${ }^{7,14}$ mostly ascribed to inadequate cutting of the sphincter, poor detrusor contractility and non-opening of the bladder-neck. It has therefore been recommended to extend sphincterotomy incisions into the bladder-neck. ${ }^{3}$ There has been some concern that bladder-neck incisions, along with sphincterotomy, may lead to constant dribbling of urine. The incidence of detrusor bladder-neck dyssynergia has been reported to be about $75 \%$. $^{6}$ Those patients with lesions above T5-6 also suffer from significant autonomic dysreflexia. It therefore seems logical to cut the bladder-neck adequately in all of these patients. A logical approach, therefore, would be to make the sphincterotomy incision at 12 o'clock from the verumontanum to the bulbous urethra and to make a 3 and 9 o'clock incision at the bladder-neck from the verumontanum to the bladder-neck. It is our experience that bladder-neck incisions are made best when the bladder contains about $150 \mathrm{ml}$ of fluid. When bladder-neck incisions are made with an empty bladder, they end up extending into the bladder and lead to unnecessary cutting of the bladder wall. With a very full bladder, the incisions do not adequately extend into the bladder-neck and a portion of the bladder-neck remains uncut.

The use of a contact laser with a crystal tip provides a safer and effective surgery as is shown with our earlier results. ${ }^{8}$ Minimal intraoperative, perioperative and secondary bleeding has been reported following laser sphincterotomy. With the use of a crystal chisel, one can make incisions like a diathermy knife until an adequate depth is accomplished and most of the external sphincter has been ablated. The excessive blood loss in our first two patients was due to the use of higher wattage which is less hemostatic and cuts through more of the vascular tissue. It is therefore important to use optimal wattage according to what is desired during surgery. If there is an opened venous sinus or while cutting through the bulbous urethra, wattage will need to be lowered to 25 or even less to control the bleeding. To begin with, we use 35 watts and when the incision is extended close to the bulbous urethra, lower wattage, preferably $20-25$ watts setting, is optimal. A higher wattage (30 to 40 watts) is required to cut the strictured area following previous electrocautery sphincterotomy. A lower wattage easily coagulates the segment of the exposed vein, whereas electrocautery produces a bigger hole in the exposed vein. The use of the contact laser virtually eliminates 'flame hemorrhages' which occur when venous sinuses are cut with electrocautery. The contact laser coagulates the whole exposed vein and this easily stops venous bleeding.

Our overall success rate of $92 \%$ with a mean followup for 27 months, is gratifying. Our four failures were mostly due to inadequate surgery during a phase when we were trying to develop the technique. Two out of three later failures were again due to not enough cutting in patients with areflexic bladders. They needed bladder-neck incisions to improve voiding. There was an increase in voiding pressures in three patients following surgery. This was a quick rise and drop and not a sustained rise in pressure as is often noticed with detrusor hypertrophy associated with outflow obstruction. It seems appropriate to open the bladder-neck with two deep lateral incisions, and also a 12 o'clock incision, in patients with an areflexic bladder to prevent repeat surgery.

Our newly developed technique of using buttonholes and the cutting of overlying tissues with a contact crystal chisel laser probe significantly reduces the time spent during surgery and results in minimal to nil bleeding. This technique provides a broader use of the laser for endoscopic surgery, when compared with the free beam laser, which can be used for prostate surgery alone; the immediate results achieved with the contact laser are an additional advantage. There is no risk of hyponatremia, and the postoperative catheter is usually required for only 1 day, when the patient can be discharged home within the first $24 \mathrm{~h}$. This early discharge is possible because there is very little chance of secondary hemorrhage following laser endoscopic surgery.

\section{Acknowledgement}

Vickie Wolfe, R.N. for helping with the urodynamics and data collection.

\section{References}

1 Brindley GS. The first 500 patients with sacral root stimulator implants. Paraplegia 1994; 32: $795-805$.

2 Chancellor MF et al. Multicentral trial in North America of UroLume urinary sphincter prosthesis. J Urol 1994; 152: $924-$ 930 .

3 Perkash I. Detrusor-sphincter dyssynergia and dyssynergic responses: recognition and rationale for early modified transurethral sphincterotomy in complete spinal cord injury lesions. $J$ Urol 1978; 120: $469-474$

4 Dykstra DD et al. Effects of botulinum A toxin on detrusorsphincter dyssynergia in spinal cord injury patients. J Urol 1988; 139: $919-922$

5 Perkash I. Modified approach to sphincterotomy in spinal cord injury patients: Indications, technique and results in 32 patients. Paraplegia 1976; 13: 247-260. 
6 Schurch B, Yasuda K, Rossier AB. Detrusor bladder neck dyssynergia revisited. J Urol 1994; 152: 2066-2070.

7 Lockhardt JL, Vorstman B, Weinstein D, Politano VA. Sphincterotomy failure in neurogenic bladder disease. $J$ Urol 1986; 135: 86-89.

8 Perkash I. Sphincterotomy and ablation of prostate using crystal chisel contact top firing neodymium: YAG laser. J Urol 1994; 152: 2020 - 2024.

9 Perkash I. Principles of Modern Urodynamic Studies. Reprinted from Investigative Radiology 1987; 22: $279-289$.

10 Perkash I, Friedland GW. Transrectal ultrasonography of the lower urinary tract: Evaluation of bladder neck problems. Neurourology and Urodynamics 1986; 59: $453-455$.
11 Ross JC, Damanski M, Gibbon N. Resection of the external urethral sphincter in the paraplegic - preliminary report. Transactions of the American Association of Genito-Urinary Surgeons 1957; 49: $193-198$.

12 Ross JC, Gibbon NOK, Damanski M. Division of the external sphincter in the treatment of the neurogenic bladder. A ten-year review. British Journal of Surgery 1967; 54: 627-628.

13 Quesada EM, Scott FB, Cardus D. Functional classification of neurogenic bladder dysfunction. Arch Phys Med 1968; 49: $692-$ 697.

14 Vapnck JM, Couillard DR, Stone AR. Is sphincterotomy the best management of the spinal cord injured bladder? J Urol 1994; 151: $961-964$. 